Cruz Colmenero, Verónica; Caballero García Presentación; Ruiz Tendero, Germán (2013). La dramatización cómó recurso didáctico para el desarrollo emocional. Un estudio en la etapa de educación primaria. Revista de Investigación Educativa, 31 (2), 393-410.

http://dx.doi.org/10.6018/rie.31.2.164501

\title{
LA DRAMATIZACIÓN COMO RECURSO DIDÁCTICO PARA EL DESARROLLO EMOCIONAL. UN ESTUDIO EN LA ETAPA DE EDUCACIÓN PRIMARIA
}

\author{
Verónica Cruz Colmenero(1), Presentación Caballero García ${ }^{(1)}$ y Germán Ruiz Tendero(2) \\ (1) Universidad Camilo José Cela de Madrid \\ (2) Universidad Complutense de Madrid
}

\section{RESUMEN}

Nuestro propósito fue comprobar los efectos de la dramatización en la expresión y reconocimiento de emociones. El estudio se realizó con 45 alumnos de primaria de ambos sexos, de 10 y 11 años de edad, divididos en dos grupos: experimental (23 alumnos) y control (22 alumnos). La recogida de datos se realizó mediante escalas de observación diseñadas ad hoc de acuerdo a los objetivos que se aplicaron antes y después de una intervención basada en la dramatización en el grupo experimental, y al mismo tiempo en el grupo control con la diferencia de que este último no recibió tratamiento. El programa de intervención constó de seis sesiones de una hora de duración en las que se trabajó la expresión y el reconocimiento de las emociones a través de ejercicios dramáticos. Los resultados demostraron que el programa mejoró significativamente la capacidad de expresión y reconocimiento de emociones de los alumnos.

Descriptores: Dramatización, Expresión facial de las emociones, reconocimiento de emociones, educación emocional.

\footnotetext{
Correspondencia:

Presentación A. Caballero García, Universidad Camilo José Cela. Facultad de Ciencias Sociales y de la Educación. Instituto de Enseñanza y Aprendizaje. Castillo de Alarcón, 49. Villafranca del Castillo. 28692 Madrid. Tlf: 91/8153131. E-mail: pcaballero@ucjc.edu
} 


\section{DRAMATIZATION AS A TEACHING RESOURCE FOR EMOTIONAL DEVELOPMENT IN PRIMARY EDUCATION}

\section{ABSTRACT}

The aim of this study was to analyze the effects of dramatization when expressing and recognizing emotions. The study was carried out with 45 male and female primary school pupils aged 10 and 11 divided into two group: experimental, 23 pupils; and control, 22 pupils. Data collection was undertaken using observation scales created ad hoc in line with research objectives, and administered before and after an educational intervention based on dramatization in the case of the experimental group. No treatment was given to the control group. The intervention program for the experimental group consisted of six one-hour sessions in which drama exercises tackled recognition and expression of emotions. The results evidenced a significant improvement in the ability to recognize and express emotions of those pupils who underwent the intervention.

Keywords: Dramatization, facial expression of emotions, emotion recognition, emotional education.

\section{INTRODUCCIÓN}

Muchos de los problemas con que se encuentran las personas, y en particular los adolescentes y jóvenes de hoy, tienen que ver con el analfabetismo emocional (Bisquerra, 2001). El analfabetismo emocional tiene unos efectos altamente negativos, no sólo sobre las personas sino también sobre las sociedades (Goleman, 1995).

La educación emocional surge como una respuesta a estas necesidades sociales que no quedan suficientemente atendidas en la educación formal (Álvarez, Bisquerra, Fita, Martínez y Pérez, 2000; Bisquerra, 2003). Es una forma de educar para la vida (personal, social, familiar, profesional, etc.). Posibilita un mayor bienestar subjetivo, mayores dosis de felicidad, y esto redunda en un mayor bienestar social (Bisquerra, 2001). Propone el desarrollo de conocimientos y habilidades sobre emociones, con objeto de capacitar al individuo para afrontar los retos que se plantean en la vida cotidiana (Bisquerra, 2000: 251).

En un momento en que muchos niños parecen tener dificultades con el manejo de sus problemas, de prestar atención o de concentrarse, de controlar sus impulsos, de sentirse responsables por su trabajo o de interesarse en su aprendizaje, cualquier cosa que sostenga estas habilidades ayudará a su educación, y justificará la necesidad de una educación emocional en la escuela. La alfabetización emocional no hace sino mejorar la capacidad que tiene la escuela para enseñar (Caballero García, 2009; Güell Barceló y Muñoz Redón, 2000; Ibarrola, 2003; Rodríguez Garza, 2005).

Sería positivo ir enseñando progresivamente a niños y niñas a poner nombre a las emociones básicas, a captar los signos de expresión emocional de los demás, a interpretarlas correctamente, asociarlas con pensamientos facilitadores de actuaciones adecuadas, y a controlarlas (Cuadrado, 1998). La formación intelectual por sí sola no se sostiene si no se complementa con el desarrollo de hábitos adecuados de conoci- 
miento emocional, expresión y comprensión de emociones, automotivación, habilidades interpersonales básicas para su equilibrio social, y técnicas de autocontrol del comportamiento (Caballero García, 2009).

La escuela de hoy está haciendo grandes esfuerzos porque esta formación integral no quede en papel mojado y sea una realidad diaria en el currículo oficial y vivido en todos sus elementos. Por eso, cualquier planteamiento curricular de las emociones deberá tener en cuenta los objetivos, los contenidos, las competencias, las estrategias metodológicas y la evaluación en su conjunto.

Torres (2000) aborda todos estos aspectos, señalando unos objetivos dirigidos a la autorrealización, a las relaciones con el grupo de convivencia, al trabajo y otros de orientación social. Como contenidos básicos señala la conveniencia de desarrollar la autoestima y la conciencia de bienestar, a los que añade los propuestos por Bisquerra (2000): conciencia de las propias emociones, control emocional, autoestima, comunicación afectiva y efectiva, relaciones interpersonales, resolución de conflictos, habilidades de vida y calidad de vida. Nuestra propuesta parte de la idea señalada por Bisquerra (2001), respecto de que la educación emocional debe integrarse en el currículum como si se tratase de un tema transversal, ajustándose a las lecciones específicas de las diversas áreas académicas, introduciéndose en un determinado curso, siendo retomado y ampliado en cursos posteriores, así como desarrollado a lo largo de toda la vida del individuo.

La enseñanza de emociones depende más del entrenamiento y su perfeccionamiento que de la instrucción verbal (Fernández-Berrocal y Extremera, 2002). Una parte importante del aprendizaje se realiza a través del ejemplo y de la imitación. Queramos o no, estamos transmitiendo un determinado modelo de gestión de las emociones que tendrá consecuencias duraderas para la vida emocional de nuestros alumnos. Se trata de que seamos conscientes de ello y que elaboremos un modelo positivo y enriquecedor en esta línea (García Moriyón, 1999).

Numerosos estudios destacan los beneficios de la gestión inteligente de las emociones. Fernández-Abascal (1997) afirma que, tanto la expresión de emociones como su inhibición, son fundamentales en el ajuste psicológico y en la salud de las personas. Un proceso de inhibición constante puede resultar patológico para éstas, mientras que la expresión de emociones es un ejercicio beneficioso, no solamente a nivel emocional sino también terapéutico.

La expresión facial es la más breve de las señales emocionales. Nowicki y Searcy (2005) comprobaron que los niños que no pueden expresar sus emociones o leer adecuadamente las de los demás se sienten continuamente frustrados. Son niños que no comprenden lo que está ocurriendo porque no llegan a acceder al subtexto constante que encuadra todo tipo de comunicación.

Las emociones básicas poseen una expresión facial diferenciada, universal y fácil de identificar. Ekman (2003) enumeró los beneficios de estar atento a nuestras respuestas emocionales: En primer lugar, porque aprenderemos más sobre las sensaciones corporales características de cada emoción, convirtiéndose éstas en señales de alerta que nos mantienen atentos a nuestros estados emocionales. En segundo lugar, porque nos ayudarán a observar mejor los sentimientos emocionales de otras personas y, en tercer lugar, porque esto lleva implícito el autocontrol, ya que disminuyendo las señales 
del rostro y de la voz, estamos controlando voluntariamente los músculos faciales que involuntariamente expresan la emoción y nos hacen que la sintamos con más intensidad. Es lo que Tomkins (1963) denominó hipótesis del feedback facial. Realizar los gestos característicos de la emoción facilita la aparición de otras variables asociadas a la misma, como el tono de voz, la postura y los pensamientos o sensaciones que están presentes en dicha reacción afectiva.

Por otro lado, también existen una serie de condicionantes para la inhibición de la expresión emocional como son: 1) Un contexto próximo que castiga o no refuerza la expresión de emociones, específico de la vivencia de cada uno (familia como entorno más cercano), en el que el niño carece de esa referencia emocional para orientarse; y 2) un contexto amplio o una sociedad construida basándose en unos valores y principios que se alejan de un plano emocional o vivencias experimentadas en la infancia y la adolescencia inhibidoras. Estos condicionantes constituyen momentos críticos para el aprendizaje, sobre los que se construyen las primeras tendencias a responder de una manera constante a ciertas dimensiones de la realidad. A lo aprendido en esas etapas se va a responder con más sensibilidad, Ekman (2005) lo llamó base de datos de alerta emocional, configurada en parte por la biología, mediante la selección natural, y en parte por la experiencia individual. A este recurso emocional se le unen lo que él también denominó reglas de exposición, hábitos motores aprendidos por normas socioculturales que sirven para modular la expresión facial o incluso para ocultar una emoción determinada y manifestar otra. Estas reglas de exposición sólo aparecen en interacciones sociales. El disimulo de las exhibiciones emocionales, además del desarrollo de exhibiciones no emocionales, está ligado al entrenamiento o a la tradición perteneciente a una determinada cultura (Ekman y Friesen, 1978). Las normas culturales acerca de las exhibiciones pueden bloquear o anular una expresión facial o enmascarar o disimular o suplantar a un semblante emocional. Es por esto que el reconocimiento de las emociones, a pesar de su expresión universal, se hace más complejo.

Respecto al reconocimiento de las emociones, aún se desconoce si es una capacidad innata o se va aprendiendo a lo largo de los años. Lo que sí se ha demostrado es que puede verse dañada por experiencias tempranas perturbadoras. Los niños abandonados o maltratados no reconocen las distintas expresiones faciales de las emociones con tanta exactitud como los niños bien tratados (Bugental, Shennum, Frank y Ekman, 2000).

Reconocer ajustadamente las emociones en los demás es importante por los siguientes motivos: Las señales emocionales que emiten los demás, a menudo determinan nuestra forma de interpretar sus palabras y acciones. Además, la expresión emocional del interlocutor provoca nuestra propia respuesta emocional, lo cual influye a su vez en nuestra interpretación de lo que la persona dice y en lo que pensamos sobre sus motivaciones, actitudes e intenciones (Ekman, 2003).

Chóliz y Fernández-Abascal (2001) enumeraron los principales factores que afectan al reconocimiento de la expresión facial de emociones: El estado emocional del observador (tanto el reconocimiento de la cualidad emocional como el de la intensidad de la misma suele ser congruente con la reacción afectiva y el nivel de activación de quien observa dicha emoción); la influencia del contexto (suministra información acerca del estado emocional, facilitando el análisis aislado de los gestos faciales); el feedback de la ejecución (reconocer es una habilidad que se aprende; durante el aprendizaje, el 
feedback que se reciba es relevante); la imitación y el modelado (el reconocimiento de emociones se optimiza cuando imitamos la emoción que vemos, puesto que nos facilita la decodificación) o las expectativas y atribuciones acerca del estado emocional (tener conocimiento previo sobre la forma de ser de alguien o sobre las circunstancias que concurren en él, que nos proporcionan indicios de lo que puede sentir una persona en un momento determinado) y por último, las diferencias individuales (tanto en la forma como manifestamos nuestras emociones como en la precisión en la identificación de las de los demás).

Las emociones son impulsos para actuar, programas de actuación que se ponen en marcha automáticamente ante determinados estímulos externos y están modelados por nuestra experiencia de vida y nuestra cultura (Goleman, 1995). Pueden ser innatas (básicas, fundamentales o primarias) o adquiridas (secundarias o aprendidas por influencia del ambiente). Nuestro trabajo se ha centrado en las primeras y en la posibilidad de educar su expresión y reconocimiento mediante la dramatización. Las emociones fundamentales, básicas o primarias poseen, según Izard (1972): a) un sustrato neural específico e innatamente determinado, b) un patrón neuromuscular expresivo característico y c) una cualidad subjetiva o fenomenológica definida.

Cada emoción presenta cuatro tipos de efectos: el efecto subjetivo, que se refiere al placer o displacer que se desprende de la situación; el efecto fisiológico, que son los cambios que se producen en el sistema nervioso central, periférico y sistema endocrino; el efecto funcional, que son los cambios comportamentales y, por último, el efecto social, que se refiere a la comunicación y exteriorización de las emociones mediante la expresión facial y otra serie de procesos de comunicación no verbal, como los cambios posturales o la entonación. A su vez, cada emoción tiene tres funciones fundamentales (Chóliz y Fernández-Abascal, 2001): la función adaptativa (prepara al organismo para que ejecute eficazmente la conducta exigida), la función motivacional (la emoción energiza la conducta motivada) y la función social (la expresión de emociones permite predecir el comportamiento asociado a las mismas, lo cual tiene un gran valor en los procesos de relación interpersonal). El efecto social de la emoción ha sido otro de nuestros focos de interés. Las exhibiciones faciales constituyen herramientas que tienen como meta la negociación en los encuentros sociales (Smith, 1977).

El valor comunicativo de la emoción es importante a la hora de tener unas relaciones sociales estables. Cuanto mejores emisores y receptores de emociones seamos, más hábiles socialmente seremos. Encuadrar este desarrollo dentro de la escuela sería fundamental y un aspecto básico de nuestra propuesta.

La expresión facial es el componente de la emoción que se manifiesta externamente con mayor claridad y congruencia y el vehículo principal de comunicación de los estados afectivos en el ser humano (Chóliz y Fernández-Abascal, 2001). Las emociones se expresan más a través de nuestro lenguaje no verbal. Pocas veces las expresamos verbalmente, hablamos de ellas, pero nuestro cuerpo está comunicando continuamente la emoción que estamos sintiendo, querámoslo o no.

Darwin (1960-1980) fue el primero en relacionar rostros-tipo con "emociones básicas", buscando una evidencia de la continuidad entre humanos, primates no humanos y no primates. El modelo neurocultural desarrollado por Ekman (1972), derivado de Darwin, que hemos utilizado como referencia para nuestro programa de intervención, 
tiene sus raíces en dos ideas principales: la existencia de emociones discriminables en los movimientos faciales y de una modulación aprendida de las exhibiciones emocionales (Tomkis, 1963), y la existencia de unos estímulos clave que liberan la emisión de secuencias de conducta motora, entre las que se incluyen las exhibiciones expresivas, consideradas secuencias innatas, cuyos componentes podrían verse ampliados o atenuados por las convenciones sociales (Fridlund, 1999).

Ekman (1972) defendió la existencia de una programación del afecto facial, localizado desde el nacimiento en el sistema nervioso, que enlaza cada emoción con un conjunto de impulsos neurales y de patrones característicos de impulsos dirigidos a los músculos faciales (p. 216). Este programa neural, cuando se activaba, normalmente inducía a la contracción de una configuración fija y parcialmente innata de los músculos faciales, gracias a un mecanismo que almacenaba los patrones que regían esta compleja organización de respuestas que, al ser activado, controlaba su aparición (Ekman y Davidson, 1994). Los patrones faciales equivalían categóricamente a cada una de las emociones primarias.

Sin embargo, aunque estas emociones fundamentales o emociones básicas constituían programaciones innatas, Ekman (1972) consideraba que no eran inmunes a la modificación, ya que el hecho de que existieran mecanismos de base genética para las emociones fundamentales, no implicaba que algún aspecto de la emoción no pudiera ser modificado. La educación, nuestra experiencia y el temperamento podían modificar nuestra forma de expresión innata.

Ekman $(1972,2003)$ encontró porcentajes muy altos de coincidencia, tanto en la expresión como en el reconocimiento de las 6 emociones básicas (alegría, asco, sorpresa, tristeza, cólera y miedo). Sin embargo, casi todo el mundo puede aprender a inhibir o modificar las expresiones faciales innatas (...). Además de aprender modificaciones de las expresiones de la emoción, las influencias socioculturales y las experiencias personales pueden representar un importante papel en la determinación de la emoción que va a emitir y que hará esa persona como resultado de esa emoción (Izard, 1991: 19).

La infancia es el período en el que se observa una mayor concordancia entre el estado emocional y su expresión facial (Izard y Malatesta, 1987). En el curso del desarrollo, el sistema nervioso se hace cada vez más "corticalizado", las exhibiciones se hacen gradualmente más sutiles y más socializadas. El resultado es que en la interacción social normal, las expresiones involuntarias de la emoción pueden ser modificadas para satisfacer las demandas de la cultura. Se disfrazan, se sofocan o intensifican de forma histriónica (Ekman y Scherer, 1984).

Ekman (1972) dio a conocer la existencia de otras emociones, además de las primarias, que incluía el modelo Neurocultural. Propuso que estas emociones y sus rostros asociados representaban emociones secundarias o emociones combinadas, cuando se evocaba más de una emoción de forma concurrente. Las emociones, en su punto culminante, podían ser interpretadas y percibidas por los demás fácilmente; sin embargo, había expresiones de ellas más sutiles, difíciles de captar y de reconocer, que indicaban que la emoción se estaba empezando a sentir, o era una emoción reprimida, o de baja intensidad.

Generalmente, estamos tan pendientes de la comunicación verbal, que nos olvidamos de señales no verbales que indican lo que siente verdaderamente la persona. El Sistema de Codificación de Acción Facial (FACS) analiza todas estas emociones, siendo 
el procedimiento de codificación de la expresión facial de las emociones más conocido y exhaustivo. Desde que Ekman y Friesen (1978) lo desarrollaran en los años setenta ha servido de fundamento a un gran número de investigaciones sobre el tema. Se trata de un procedimiento que permite identificar las emociones en base a la actividad muscular implicada en los gestos faciales.

Entre los muchos recursos metodológicos que pueden emplearse para la educación de las emociones y su expresión, Torres (2000) recomienda las técnicas creativas y dramáticas. Precisamente son estas expresiones sutiles de las emociones las que se entrenan durante la actividad dramática, tanto expresándolas como reconociéndolas, con el objetivo de contribuir al desarrollo de la competencia emocional. Este planteamiento se convirtió en un reto para la realización de este trabajo.

En nuestra revisión bibliográfica del tema encontramos que la dramatización se ha utilizado con fines educativos en diferentes contextos, edades y grupos humanos (Navarro Solano, 2009). Goza de amplia experiencia en diversos sistemas educativos europeos, destacando el caso del Reino Unido. Sin embargo, en nuestro país, ha tenido un uso educativo en general escaso y poco reglado. A ello se le une la poca tradición investigadora en esta área. Todo ello hace que, a pesar de las evidencias experimentales sobre las capacidades que la dramatización posee de cara a la educación emocional (Navarro Solano 2005; Motos 2003), los trabajos de investigación sobre este tema sean manifiestamente insuficientes (Navarro Solano, 2009).

La práctica dramática mejora el conocimiento de nosotros mismos y favorece a su vez la comunicación interpersonal e intergrupal (Navarro Solano, 2007), dos habilidades íntimamente relacionadas con el concepto de inteligencia intrapersonal e interpersonal (Gardner, 1998). Sin embargo, la dramatización en la escuela y la educación emocional son asignaturas aún pendientes en la mayoría de los centros educativos (Cruz Colmenero, 2008). A lo largo de los años en los que hemos impartido la asignatura de Expresión Dramática en las etapas de educación infantil y primaria, hemos ido corroborando que es una materia que tiene mucho que ver con la educación emocional del alumno.

Utilizando la pedagogía teatral y el Sistema de Codificación de Acción Facial de Ekman (2003) diseñamos un programa de intervención que intentaba mejorar en los niños de primaria con los que estábamos trabajando la capacidad de expresar las emociones a nivel facial y de reconocerlas en los demás.

Hasta ahora, habíamos utilizado el FACS como un sistema que ayudaba a identificar y a reconocer las emociones en los demás, pero la novedad era utilizarlo también como base para un programa de entrenamiento que mejorara ambos aspectos de la emoción, e hiciera más hábiles a las personas que trabajaban con él, no sólo como receptores sino también como emisores. Introducirlo además dentro del currículum escolar, fue todo un reto. Los niños aprendieron a desarrollar sus emociones través del juego dramático y se convirtieron en mejores observadores y emisores de las emociones.

El objetivo de la investigación fue conocer el nivel de reconocimiento y de expresión facial de emociones de niños de 10 y 11 años, con el fin de comprobar la mejora de sus habilidades emocionales, tras someterlos a un programa de intervención basado en la dramatización. 


\section{MÉTODO}

Para dar cumplimiento a este objetivo se recurrió a un diseño cuasi-experimental pretest-postest con grupo de control no equivalente (Ary, Jacobs y Razaviech, 1982).

\section{Participantes}

La muestra estuvo formada por un total de 49 alumnos, 23 del grupo experimental (11 varones y 13 mujeres) y 22 alumnos del grupo control (12 varones y 10 mujeres), que fueron seleccionados mediante un muestreo no probabilístico intencional, respetando el sistema de grupos intactos. Los alumnos, de edades comprendidas entre los

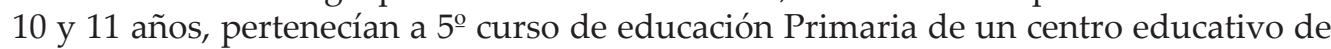
la zona noroeste de la Comunidad de Madrid, cuya población mayoritaria se incluye en un estatus socioeconómico medio-alto.

\section{Instrumentos}

Los instrumentos de recogida de información fueron tres escalas de observación (escalas A, B, y C) y una cámara de vídeo modelo DCR- SR52, Sony Handicam HDD, CDDC de 1 megapíxel, con zoom óptico de 25 aumentos, una lente Carl Zeiss y disco duro de $30 \mathrm{~GB}$, que se utilizó con un trípode para minimizar interferencias.

La escala A, de elaboración propia y basada en el estudio facial de las seis emociones básicas realizado por Ekman (1972), estuvo compuesta por 18 ítems y se preparó para ser cumplimentada por el profesor y el alumno. Evaluaba la capacidad de los niños para expresar las 6 emociones básicas (alegría, tristeza, miedo, ira, asco, sorpresa), en tres intensidades distintas (alta, media y baja), cuyo orden en el cuestionario fue aleatorio, y de acuerdo a una escala tipo Likert de 1-5, donde el 1 significaba muy mal y 5 muy bien. El profesor indicaba al niño una emoción que debía expresar; tras hacerlo, éste evaluaba su propio desempeño. Seguidamente, y manteniendo la expresión facial, el niño se miraba en un espejo y volvía a evaluarse sobre la misma escala, al igual que también lo hacía el profesor, por su parte, y en los mismos tiempos.

Las escalas B y C se utilizaron para evaluar la capacidad de reconocimiento de las emociones de los niños en los demás. En la escala B, el niño visualizaba 20 vídeos de Chóliz y Fernández-Abascal (2001), de 5 segundos de duración, con una persona cada vez distinta expresando una emoción. El niño debía rodear con un círculo la emoción básica que creía reconocer. En la escala C, el alumno debía escoger de 22 fotos extraídas del test de reconocimiento facial de Ekman (2003) y de las fotografías expuestas en su libro "¿Qué dice ese gesto?", la emoción o emociones combinadas que creía reconocer, puntualizando además si la emoción era expresada de forma total (cuando la emoción se expresa con todo el rostro) o parcial (cuando una persona intenta regular su expresión emocional para disminuir cualquier indicio).

Las escalas A y B obtuvieron buenos índices de consistencia interna según el modelo Alfa de Cronbach, tanto en el pretest como en el postest (tabla 1). Por su parte la escala $\mathrm{C}$ fue la que originó valores de consistencia más bajos, considerándose su fiabilidad 
como moderada según los valores de referencia ofrecidos en la literatura (Salvucci, Walter, Conley, Fink y Saba, 1997).

TABLA 1

FIABILIDAD DE LAS ESCALAS SEGÚN MODELO ALFA DE CRONBACH

\begin{tabular}{|c|c|c|c|c|c|}
\hline & $\begin{array}{c}\text { Escala A } \\
\text { (evaluación } \\
\text { del profesor) }\end{array}$ & $\begin{array}{c}\text { Escala A } \\
\text { (evaluación } \\
\text { en espejo) }\end{array}$ & $\begin{array}{c}\text { Escala A } \\
\text { (evaluación } \\
\text { del alumno) }\end{array}$ & Escala B & Escala C \\
\hline Pretest & 0,92 & 0,91 & 0,87 & 0,71 & 0,54 \\
\hline Postest & 0,97 & 0,93 & 0,89 & 0,75 & 0,66 \\
\hline
\end{tabular}

Para las sesiones de entrenamiento con los alumnos utilizamos la versión reducida del FACS de Ekman, propuesta por (Chóliz y Fernández-Abascal, 2001), adaptada para que pudiera ser entendida por niños de 10 y 11 años.

\section{Procedimiento}

Establecido el primer contacto con el centro y aprobada por el equipo directivo la participación en el estudio, se escogieron dos grupos de alumnos de $5^{\mathbf{o}}$ de primaria, uno de ellos asignado al azar como grupo control y otro como grupo experimental.

La aplicación de los registros de observación sistemática se realizó en el tercer trimestre, en horario lectivo de la asignatura de Expresión Dramática y en dos momentos del tiempo: antes y después del entrenamiento para el desarrollo de las emociones, en el caso del grupo experimental, y al mismo tiempo, en el caso del grupo control, con la diferencia de que éste último no recibió tratamiento alguno.

Previo a la evaluación, se realizaron dos ensayos con cada alumno para la familiarización con el sistema y la videograbación. La evaluación se llevó a cabo en el gimnasio del centro, el cual disponía de un espejo.

En la administración de la escala A, el profesor indicaba al niño la emoción, éste la expresaba, el profesor la evaluaba y luego le pedía que se mirara en un espejo y valorara cómo la había desarrollado del 1 al 5, donde 1 significaba muy mal y 5 muy bien. En la administración de las escalas B y C, al niño se le daban unas instrucciones para que reconociera emociones en los demás y las identificara por escrito. En la escala B, el niño visualizaba 20 vídeos de cinco segundos de duración que contenían emociones básicas; en la escala $C$, escogía entre 22 fotos las emociones básicas y combinadas que creía reconocer. La sesión terminaba con un intercambio de impresiones entre profesora y alumnos acerca del resultado la experiencia.

La fase de tratamiento que recibió el grupo experimental fue dirigida por la profesora de la asignatura de Expresión Dramática y consistió en seis sesiones de una hora de duración, una por cada emoción básica (alegría, tristeza, sorpresa, ira, asco y miedo), en las que los niños desarrollaron, de manera individual y por parejas, sus capacidades de reconocimiento y expresión emocional. Las sesiones se desarrollaron siguiendo el siguiente esquema: 1) motivación hacia los contenidos e introducción a los objetivos de la sesión, 2) desarrollo de actividades propias de la pedagogía teatral 
para la expresión y el reconocimiento emocional, tales como expresión corporal, mimo, maquillaje, cuentacuentos, clown e improvisaciones, en cuyo caso, y dependiendo de las mismas se optó por una organización del grupo-clase en parejas o individual, y 3) puesta en común e intercambio de impresiones en gran grupo.

\section{Análisis de datos}

El análisis de datos se llevó a cabo mediante el paquete estadístico SPSS (versión, 19). Para analizar las diferencias entre el pretest y el postest se empleó la prueba $T$ de Student para muestras relacionadas, estableciéndose un nivel de confianza igual o superior al 95\%. Previo a los análisis se comprobó que las variables analizadas de cada escala se ajustaban a la normalidad de la población, mediante el test KolmogorovSmirnov para una muestra. Para el cálculo de la consistencia interna de las diferentes escalas se hallaron los valores de $\alpha$ de Cronbach.

\section{RESULTADOS}

En la tabla 2 se presentan los resultados de la intervención para las tres escalas empleadas. Respecto a la escala A de expresión de las emociones, observamos que el grupo experimental (GE) mejoró significativamente en el promedio de la escala $(<, 001)$, cuando la evaluación fue llevada a cabo por el profesor $(\mathrm{p}<, 05)$ y el alumno se evaluó en el espejo $(<, 001)$. Respecto a la evaluación del propio alumno sobre la expresión de las emociones también hubo mejora, pero el contraste estadístico no le otorgó signi-

TABLA 2

EFECTO DE MEJORA EN LAS TRES ESCALAS EMPLEADAS

\begin{tabular}{|c|c|c|c|c|c|c|}
\hline & \multicolumn{2}{|c|}{ Grupo control } & \multicolumn{2}{|c|}{ Grupo Experimental } \\
\hline & & & Pretest & Postest & Pretest & Postest \\
\hline \multirow{8}{*}{$\begin{array}{c}\text { Escala } \\
\text { A }\end{array}$} & \multirow{2}{*}{$\begin{array}{l}\text { Eval } \\
\text { profesor }\end{array}$} & $X( \pm S D)$ & $3,06(0,72)$ & $2,89(0,64)$ & $3,21(0,71)$ & $4,12(0,82)$ \\
\hline & & Dif & \multicolumn{2}{|c|}{ * } & \multicolumn{2}{|c|}{ * } \\
\hline & \multirow{2}{*}{$\begin{array}{l}\begin{array}{l}\text { Eval } \\
\text { alumno }\end{array} \\
\end{array}$} & $\mathrm{X}( \pm \mathrm{SD})$ & $3,39(0,52)$ & $3,35(0,52)$ & $2,95(0,50)$ & $3,19(0,58)$ \\
\hline & & Dif & \multicolumn{2}{|c|}{ (1) } & \multicolumn{2}{|c|}{ 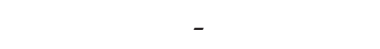 } \\
\hline & \multirow{2}{*}{$\begin{array}{l}\text { Eval } \\
\text { espejo }\end{array}$} & $X( \pm S D)$ & $3,38(0,91)$ & $3,46(0,58)$ & $2,96(0,45)$ & $3,34(0,55)$ \\
\hline & & Dif & & \multicolumn{2}{|c|}{$* * *$} \\
\hline & \multirow{2}{*}{$\begin{array}{l}\text { Promedio } \\
\text { escala A }\end{array}$} & $\mathrm{X}( \pm \mathrm{SD})$ & $3,33(0,45)$ & $3,23(0,45)$ & $3,04(0,40)$ & $3,55(0,45)$ \\
\hline & & Dif & \multicolumn{2}{|c|}{ * } & \multicolumn{2}{|c|}{$* * *$} \\
\hline \multirow{2}{*}{$\begin{array}{c}\text { Escala } \\
\text { B } \\
\end{array}$} & & $\mathrm{X}( \pm \mathrm{SD})$ & $15,05(1,64)$ & $15,36(2,68)$ & $15,83(2,88)$ & $14,65(2,42)$ \\
\hline & & Dif & & \\
\hline \multirow{2}{*}{$\begin{array}{c}\text { Escala } \\
\text { C }\end{array}$} & & $\mathrm{X}( \pm \mathrm{SD})$ & $11(2,61)$ & $14,25(2,75)$ & $12,82(2,64)$ & $16,82(3,81)$ \\
\hline & & Dif & \multicolumn{2}{|c|}{$* * *$} & \multicolumn{2}{|c|}{$* * *$} \\
\hline
\end{tabular}

(Dif: diferencias; $\left.{ }^{*}: \mathrm{p}<0,05 ;{ }^{* *}: \mathrm{p}<0,01 ;{ }^{* * *}: \mathrm{p}<0,001\right)$ 
ficación. Por su parte el grupo control (GC) no obtuvo mejora alguna en ninguno de los aspectos evaluados.

En la escala B, ambos grupos (experimental y control) mejoraron en el reconocimiento de las emociones proyectadas en video, aunque no podemos considerar esta mejora como significativa. No obstante, el GE obtuvo una mejora de 0,5 puntos superior al GC.

En la escala $C$ de reconocimiento de emociones sobre fotos, comprobamos que ambos grupos mejoraron significativamente $(\mathrm{p}<0,001)$, siendo mayor la del GE (4 aciertos más de media, frente a los 3,25 del GC).

\section{DISCUSIÓN}

El presente trabajo de investigación ha constatado que un entrenamiento basado en la dramatización mejora el nivel de expresión y reconocimiento de las emociones. Investigaciones actuales avalan la importancia de favorecer el desarrollo emocional debido a sus múltiples beneficios: mejora la capacidad de identificación de las emociones (Fellner et al., 2007) y el rendimiento académico (Weissberg, 2002), facilita una mejor comunicación interpersonal (Ekman, 2003), favorece el contagio emocional (Rizzolatti y Sinigaglia, 2006) y la empatía (Caravita, Di Blasio y Salmivalli, 2009; Gini, Albiero, Benelli y Altoè, 2007; Rizzolatti y Sinigaglia, 2006), el autocontrol y la regulación de nuestras emociones (Ekman, 2003). El desarrollo socio-afectivo se considera un elemento indisociable de la formación integral de los alumnos (Murillo y Hernández-Castilla, 2011). La neurobiología apoya estos beneficios al indicar una relación directa entre expresión, llegando incluso a la afirmación "si no expresamos, no sentimos" (Damasio, 2003), por tanto, expresar ajustadamente las emociones favorece una buena salud física y emocional.

En los últimos años múltiples investigaciones están demostrando los efectos positivos de los programas de inteligencia emocional en la escuela (Aritzeta y Gartzia, 2009; Diekstra, Sklad, Gravestijn, y Ritter, 2008; Durlak, Weissberg y Pachan, 2010; Durlak, Weissberg, Dymniki, Taylor y Schellinger, 2011; Extremera y Berrocal, 2003; Melero y Palomera, 2011, etc.) y, más específicamente, los desarrollados con alumnos de 10 y 11 años, como muestran los resultados del estudio de Caballero et al., (2012), Extremera y Berrocal (2004), Fundación Botín (2008, 2011), o los estudios de Sotil et al. (2008), entre otros.

Uno de los propósitos de esta investigación ha sido sensibilizar a los educadores y los centros educativos sobre la importancia de la educación explícita de las emociones, y de los beneficios personales y sociales que esta educación conlleva (De Pablos, Colás y González, 2011). Otra de las finalidades del estudio ha sido encontrar un modelo de entrenamiento fácil de llevar a cabo por los docentes. Nuestra postura, en consonancia con la del modelo de inteligencia emocional de Mayer y Salovey (1997), es la de ejercitar y practicar las capacidades emocionales de expresión y reconocimiento de emociones a nivel no verbal. Estas capacidades son innatas, pero convertirlas en habilidades a través del refuerzo que proporciona un programa de entrenamiento, fortalece este desarrollo y nos hace más inteligentes emocionalmente.

El lenguaje dramático es un vehículo imprescindible para trabajar el desarrollo emocional (Núñez Cubero y Romero Pérez, 2009; Ruiz de Velasco, 2000). Los resulta- 
dos del trabajo de investigación que presentamos han demostrado que con un número breve de sesiones de intervención basadas en la dramatización, los alumnos mejoran el reconocimiento y la expresión de las emociones. Son datos que también constataron autores como Abarca (2003), Núñez Cubero y Navarro Solano (2007), Navarro Solano (2005), Moreno (1999) y Motos (1992).

Núñez Cubero y Romero Pérez $(2004,2009)$ describieron los beneficios que aporta la dramatización al desarrollo de la educación emocional en estudiantes de bachillerato y de enseñanza universitaria. Cutillas (2005) sugiere la enseñanza de la dramatización y el teatro como propuesta didáctica para la enseñanza secundaria. La experiencia que mostramos mediante este estudio lo hace también extensible a la etapa de primaria. Datos todos estos útiles para los centros educativos que se planteen incluir dentro del currículum escolar un programa de intervención para el desarrollo emocional en esta línea.

El drama constituye un laboratorio de la realidad o un ensayo para la vida (Mato, 2006), porque sobre él volcamos parte de la realidad y ensayamos con ella, sabiendo que estamos en un espacio imaginado, y que por tanto, no va a tener consecuencias cómo ocurriría en el espacio real (Mallika, 2000). El primer paso para construir estos contextos imaginados es, precisamente, la implicación de nuestros sentimientos e imaginación, o de nuestra afectividad (Winston, 2000; Zillmann, 1994).

El drama en la educación da lugar a un aprendizaje vivencial real, no fingido. Aunque el contexto creado sea ficticio, las emociones provocadas son reales y habrá que estar muy atentos a ellas para que se lleguen a reconocer y a poder ser evaluadas. A pesar de su potencialidad, verificada en múltiples ocasiones, tanto en el plano teórico como en el práctico, la escasa formación inicial del profesorado hace que la dramatización sea una de las grandes ausentes en nuestras aulas (Navarro Solano, 2006/2007).

El drama en la educación trabaja desde una metodología que favorece la confianza, despierta el afecto y la empatía hacia el otro, descubre la importancia de los demás, experimenta con la realidad, explora las emociones y sentimientos, e intenta reflexionar sobre todo ello, transponiéndolos a contextos reales. Entre los elementos indispensables para que el drama contribuya al desarrollo de las emociones se encuentran la escucha, la confianza, la presencia lúdica, la creatividad e imaginación, el grupo y el compromiso común hacia el mismo, la realidad o la experiencia, y la transposición de la experiencia vivida (evaluación) (Navarro Solano, 2006/2007; 2009).

Pero la práctica dramática favorece igualmente la comunicación interpersonal e intergrupal (Gadner, 1998). Motos (2005) señala la asertividad como una de las aportaciones esenciales del drama a la educación emocional, unido a la escucha y la autoestima. La dramatización se muestra como un potente instrumento para el desarrollo de habilidades sociales y educación en valores, por su fuerte carácter interpersonal y relacional (Navarro Solano, 2006/2007; Núñez Cubero y Navarro Solano, 2007).

El drama ayuda a generar un clima de confianza en el grupo, que hace posible a sus miembros expresarse, comunicarse y manifestarse tal y como son, al tiempo que se sienten aceptados por él. Precisamente esa confianza en uno mismo y en los demás, es la que ayuda a cada uno de sus componentes a superar el miedo al ridículo. El juego dentro de este proceso se convierte en un elemento clave para el logro de este objetivo (Navarro Solano, 2009). En este sentido, existen diferentes experiencias a través del 
drama en educación para transformar y mejorar completamente centros escolares, en la medida que, en particular, cambian las formas de relación entre sus miembros (Dickinson, Neelands y Shenton Primary School, 2006).

Desde la actividad dramática también se aborda la enseñanza de valores (Núñez Cubero y Navarro Solano, 2007) relacionados con el respeto al otro y a la diversidad (Grady, 2000). El descubrimiento de los demás en la expresión dramática va unido al vínculo afectivo hacia los mismos, al desarrollo de la sensibilidad hacia ellos y sus situaciones particulares.

Con una formación específica en dramatización y para el desarrollo de las emociones, el docente podrá aplicar estas técnicas para favorecer el desarrollo emocional de los alumnos (Núñez Cubero y Navarro Solano, 2007), con los probados beneficios sociales y personales que esto conlleva. Proponemos un modelo educativo para el desarrollo emocional que asegure la igualdad de oportunidades (en lo que a educación emocional se refiere), respete la propia identidad, se adapte a la diversidad, huya de discriminaciones por estereotipos sociales y culturales de género, y consiga una verdadera coeducación (Caballero García, 2004).

Cabe reflexionar sobre el hecho de que, en nuestro estudio, los resultados más positivos se dan en aquellos alumnos que en el pretest tenían resultados más bajos, hecho que puede estar influenciado por una adaptación favorable al entrenamiento seguido y por tener un mayor margen de mejora individual.

La prueba $C$ resultó ser la más complicada para los sujetos, en cuanto a la probabilidad de acierto se refiere, pues no bastaba únicamente con reconocer la emoción sino también la intensidad de la emoción. Este hecho, unido a las limitaciones muestrales, probablemente haya determinado menores índices de consistencia interna de ésta frente a las otras escalas. En este caso, los dos grupos mejoraron su puntuación, no obstante estos datos han de ser interpretados con cautela por los motivos anteriormente expuestos.

La experiencia positiva con el programa llevado a cabo sugiere futuras líneas de actuación que suplementen las condiciones de investigación bajo las cuales se han llevado a cabo la intervención, como una evaluación del programa desde el punto de vista de la efectividad a través de estudios causales, como señalaron Núñez Cubero y Romero Pérez (2009), o un incremento en la precisión de los instrumentos de evaluación, de la muestra y del número de horas de entrenamiento en comunicación no verbal y verbal de las emociones.

Beelman y Lösel (2006) apuestan por extender la duración de este tipo de programas desde unos meses hasta incluso un año para lograr así una mayor consistencia en los resultados. Convendría además hacer un seguimiento de las capacidades de reconocimiento y expresión emocional del alumnado un tiempo después de haber finalizado el programa. Varios estudios sugieren que el mantenimiento de la intervención, por ejemplo a través de sesiones de refuerzo a intervalos regulares después de acabar el programa en educación emocional, es necesario para que se mantengan los beneficios a largo plazo. (Kraag, Zeegers, Kok, Hosman y Abu-Saad, 2006). 


\section{CONCLUSIONES}

Tras la aplicación del entrenamiento en expresión y reconocimiento de emociones a través de la dramatización, el grupo experimental mejoró significativamente en dos de las tres medidas llevada a cabo (A: expresión de las emociones y C: reconocimiento de emociones sobre fotos). Sin embargo, en la escala $\mathrm{C}$ obtuvimos niveles moderados de consistencia interna. Si a este dato le unimos una leve mejora del grupo de control en esta misma prueba, los resultados de esta escala deben interpretarse con cautela. Aunque el reconocimiento de emociones sobre video no logró la significación estadística, el grupo experimental obtuvo una mejor puntuación.

En general, los resultados de nuestro estudio apuntan hacia una mejora en la competencia emocional de nuestros grupos, más acentuada en el grupo experimental que el grupo control, como consecuencia del tratamiento. El programa de entrenamiento en expresión y reconocimiento de emociones a través de la dramatización se muestra efectivo en la mejora de la expresión y el reconocimiento de emociones en uno mismo y en los demás. Las competencias para el reconocimiento y la expresión de emociones están relacionadas entre sí; de manera que, el refuerzo de una redunda en el desarrollo de la otra.

Los resultados sugieren seguir trabajando por la unión de una pedagogía teatral y el entrenamiento en expresión y reconocimiento de emociones, con la dramatización como estrategia didáctica a la hora de trabajar por el desarrollo emocional de nuestros alumnos. Al mismo tiempo se hace necesario seguir investigando en este campo para apoyar los resultados encontrados y superar sus limitaciones.

\section{BIBLIOGRAFÍA}

Abarca, M. (2003). La educación emocional en la educación primaria. Currículo y práctica. Tesis doctoral. Barcelona: Universidad de Barcelona.

Álvarez, M., Bisquerra, R., Fita, E., Martínez, F. y Pérez, N. (2000). Evaluación de programas de educación emocional. Revista de Investigación Educativa, 18(2), 587-599.

Aritzeta, A. y Gartzia, L. (2009). Impacto de programas de formación en competencias emocionales en contextos educativos. En P. Fernández Berrocal, N. Extremera, R. Palomera, D. Ruiz-Aranda y J.M. Salguero y R. Cabello (Coords.). Avances en el estudio de la inteligencia emocional (p. 399-404). Santander: Fundación Marcelino Botín.

Ary, D., Jacobs, L.C. y Razaviech, A. (1982). Introducción a la investigación pedagógica. México: Interamericana.

Beelmann, A. y Lösel, F. (2006). Child Social Skills Training in Developmental Crime Prevention: Effects on Antisocial Behavior and Social Competence. Psicothema, 18(3), 603-610.

Bisquerra, R. (2000). Educación emocional y bienestar. Barcelona: Praxis.

Bisquerra, R. (2001). Orientación psicopedagógica y educación emocional en la educación formal y no formal. @gora Digit@l. Revista Científica Electrónica, 2.

Bisquerra, R. (2003). Educación emocional y competencias básicas para la vida. Revista de Investigación Educativa, 1(21), 7-43. 
Bugental, D.B., Shennum, W., Frank, M. y Ekman, P. (2000). “True Lies”: Children's abuse history and power attributions as influences on deception detection. Attribution, Communication Behavior, and Close Relationships, 248-265. Cambridge: Cambridge University Press.

Caballero García, P.A. (2004). Cómo enfocar la educación emocional dentro del aula desde la perspectiva de género. Actas de la Conferencia Internacional de Orientación, Desarrollo de la Carrera e Inclusión Social. A Coruña, 15-17 de septiembre.

Caballero García, P.A. (2009). Competencias emocionales: aprendizaje, desarrollo y evaluación. Madrid: Servicio de Publicaciones de la Universidad Camilo José Cela.

Caballero García, P.A. y Cruz Colmenero, V. (2009). La dramatización como estrategia didáctica y de aprendizaje en la expresión y el reconocimiento de las emociones. Comunicación presentada al II Congreso Internacional de Inteligencia Emocional. Santander, 16-18 de septiembre.

Caballero García, P., Punset, E., Bueno, A., Salinero, J.J., Marina, E. y Carretero, M.J. (2012). Estudio sobre el aprendizaje social y emocional en la etapa de educación primaria. Madrid: Universidad Camilo José Cela.

Caravita, S.C., Di Blasio, P., y Salmivalli, C. (2009). Unique and interactive effects of empathy and social status on involvement in bullying. Social Development, 18, 140-163.

Cruz Colmenero, V. (2008). La dramatización como estrategia didáctica para mejorar la expresión y el reconocimiento de emociones. Diploma de Estudios Avanzados. Madrid: Universidad Camilo José Cela.

Cuadrado, M. (1998). Los programas de desarrollo de la inteligencia emocional. Recuperado el 12 de noviembre de 2012, de www.waece.com/biblioteca/biblio_html/word/ref_d/ d071.doc.

Cutillas Sánchez, V. (2005). La enseñanza de la dramatización y el teatro: Propuesta didáctica para la enseñanza secundaria. Valencia: Universitat de València. Recuperado el 20 de abril de 2013, en http://www.tdx.cat/handle/10803/9625

Chóliz, M., y Fernández-Abascal, E. (2001). Expresión facial de la emoción. Madrid: UNED.

Damasio, A. (2003). En busca de Spinoza. Neurobiología de la emoción y del sentimiento (3a ed.). Barcelona: Crítica.

Darwin, C. (1984). La expresión de las emociones en los animales y en el hombre. Madrid: Alianza Editorial.

De Pablos, J., Colás, P. y González, T. (2011). Bienestar docente e innovación con tecnologías de la información y la comunicación. Revista de Investigación Educativa, 29(1), 59-81.

Diekstra, R.F.W., Sklad, M., Gravestijn, C., Ben, J. y de Ritter, M. (2008). La enseñanza de las habilidades emocionales y sociales en el mundo. Un estudio meta-analítico acerca de su eficacia. En Fundación Marcelino Botín (Ed.), Educación Emocional y Social. Análisis Internacional (pp. 299-315). Santander: Fundación Marcelino Botín.

Dickinson, R., Neelands, J. y Shenton Primary School (2006). Improve your Primary School through drama. Gran Bretaña: David Fulton Publishers.

Durlak, J.A., Weissberg, R.P. y Pachan, M. (2010). A Meta-Analysis of After school programs that seek to promote personal and social skills in children and adolescents. American Journal of Community Psychology, 45, 294-309. 
Durlak, J.A., Weissberg, R.P., Dymniki, A.B., Taylor, R.D. y Schellinger, K.B. (2011). The impact of enhancing studient's social and emotional learning: a meta-analysis of school based universal interventions. Child Development, 82, 405-432.

Ekman, P. (1972). Universals and cultural differences in facial expressions of emotion. En J. Cole (Ed.), Nebraska Symposium on Motivation, 19, 207-283. Lincoln, NE: University of Nebraska.

Ekman, P. (2003). ¿Qué dice ese gesto?. Barcelona: Integral.

Ekman, P. (2005). Cómo detectar mentiras. Madrid: Paidós.

Ekman, P. y Friesen, W.V. (1978). Facial Action Coding System. Palo Alto, CA: Consulting Psychologists Press.

Ekman, P. y Scherer, R. (1984). Approaches to emotion Hillsdale, N.J.: L. Erlbaum Associates.

Ekman, P. y Davisdon, R (1994). The nature of the emotion. New Yersey: Oxford University Press.

Extremera Pacheco, N. y Fernández-Berrocal, P. (2003). La inteligencia emocional en el contexto educativo: Hallazgos científicos de sus efectos en el aula. Revista de Educación, 332, 97-116.

Extremera Pacheco, N. y Fernández-Berrocal, P. (2004). El papel de la inteligencia emocional en el alumnado: evidencias empíricas. Revista Electrónica de Investigación Educativa, 6(2), 1-17.

Fellner, A., Matthews, G., Funke, G.J., Emo, A.K., Pérez-González, J.C., Zeidner, M. y Roberts, R. (2007). The effects of emotional intelligence on visual search of emotional stimuli and emotional identification. Proceedings of the human factors and ergonomics Society Annual Meeting 2007, 51 (14), 845-849.

Fernández-Abascal, E. (1997). Psicología general. Motivación y emoción (3aㅡ ed.). Madrid: Centro de estudios Ramón Areces.

Fernández-Berrocal, P. y Extremera, N. (2002). La inteligencia emocional como una habilidad esencial en la escuela. Revista Iberoamericana de Educación, 29, 1-6.

Fridlund, A.J. (1999). Expresión facial humana. Una visión evolucionista. Bilbao: Desclée de Brouwer.

Fundación Botín (2008). Educación emocional y social. Análisis internacional. Santander: Fundación Botín.

Fundación Botín (2011). Educación emocional y social. Análisis internacional. Santander: Fundación Botín.

García Moriyón, F. (1999). Derechos humanos y educación: textos complementarios. Barcelona: Ediciones de la Torre.

Gardner, H. (1998). Inteligencias múltiples. La teoría en la práctica. Barcelona: Paidós.

Gini, G., Albiero, P., Benelli, B. y Altoè, G. (2007). Does empathy predict dolescents' bullying and defending behavior?. Aggressive Behavior, 33, 467-476.

Goleman, D. (1995). Inteligencia emocional (62 ed. ed.). Barcelona: Kairós.

Grady, S. (2000). Drama and Diversity. A pluralistic perspective for educational drama. Portsmourth: Heinemann.

Güell Barceló, M. y Muñoz Redón, J. (2000). Desconócete a ti mismo. Programa de alfabetización emocional. Papeles de Pedagogía. Barcelona: Ediciones Paidós.

Ibarrola, B. (2003). Dirigir y educar con inteligencia emocional. Ponencia presentada en el VII congreso de Educación y Gestión, Madrid, noviembre. 
Izard, C.E. (1972). Patterns of emotion: a new analysis of anxiety and depression. New York: Academic Press.

Izard, C.E. (1991). The psicology of emotions. New York: Plenium.

Izard, C.E. y Malatesta, C.Z. (1987). Perspectives on emotional development I: Differential emotions theory of early emotional development. En J., Osofsky (Ed.) Handbook of infant development. New York: Wiley-Interscience.

Kraag, G., Zeegers, M.P., Kok, G., Hosman, C. y Abu-Saad, H.H. (2006). School Programs Targeting Stress Management in Children and Adolescents: A Meta - Analysis. Journal of School Psychology, 44, 449-472.

Mallika, H. (2000). Drama's Ways of Learning. Research in Drama Education, 5 (1), 45-62.

Mato López, M. (2006). El baúl mágico. Imaginación y creatividad con niños de 4 a 7 años. Ciudad Real: Naque.

Mayer, J.D. y Salovey, P. (1997). What is emotional intelligence?. New York: Basic Books.

Melero, Ma.A. y Palomera, R. (2011). Efectos de un Proyecto de educación Emocional y Social sobre el desarrollo infanto-juvenil: Proyecto VyVE (Vida y Valores en Educación). En Fundación Marcelino Botín (Ed.), Educación Emocional y Social. Análisis Internacional (pp. 63-81). Santander: Fundación Marcelino Botín.

Moreno Ramos, J. (1999) Didáctica del vocabulario en la E.S.O.: Estudio empírico. Tesis Doctoral. Murcia: Universidad de Murcia.Motos, T. (1992). Las técnicas dramáticas: Procedimiento didáctico para la enseñanza de la lengua y la literatura en la educación secundaria. Tesis Doctoral. Valencia: Universidad de Valencia.

Motos, T. (2003). Cerebro emocional, educación emocional y expresión corporal. Actas del Primer Congreso Internacional de Expresión Corporal (pp. 101-118). Salamanca: Amaru.

Motos, T. (2005). Expresión total y educación emocional. Recrearte. Recuperado el 2 de diciembre de 2012, en http://www.iacat.com/1-Cientifica/tomas.

Motos, T. y Navarro, A. (2003). El papel de la dramatización en el currículum. Articles, $29,10-28$.

Murillo, F.J. y Hernández-Castilla, R. (2011). Efectos escolares de factores socio-afectivos. Un estudio Multinivel para Iberoamérica. Revista de Investigación Educativa, 29(2), 407-427.

Navarro Solano, M.R. (2005). El valor pedagógico de la dramatización: su importancia en la formación inicial del Profesorado. Tesis Doctoral. Sevilla: Universidad de Sevilla.

Navarro Solano, M.R. (2006/2007). Drama, creatividad y aprendizaje vivencial: Algunas aportaciones del drama a la educación emocional. Cuestiones Pedagógicas, 18, 161-172.

Navarro Solano, M.R. (2009). Dramatización y Educación Emocional. Avances en el Estudio de la Inteligencia Emocional. Comunicación presentada al I Congreso Internacional de Inteligencia Emocional (vol. 1, pp. 545-550). Málaga: Fundación Marcelino Botín.

Nowicki, S. y Searcy, W. (2005). The evolution of animal communication: reliability and deception in signaling systems. New Yersey: Oxford University Press.

Núñez Cubero, L. y Navarro Solano, M.R. (2007). Dramatización y educación: aspectos teóricos. Teoría de la educación, 19, 225-252.

Núñez Cubero, L. y Romero, C. (2004). La educación emocional a través del lenguaje dramático. Addenda a la II Ponencia: Los lenguajes de las Artes. Escenas y Escenarios en Educación, XXII. Site-Sitges Conference. 
Núñez Cubero, L. y Romero, C. (2009). Proyecto “Eudaimon": un programa de desarrollo de la inteligencia emocional en estudiantes universitarios. En P. Fernández Berrocal, N. Extremera, R. Palomera, D. Ruiz-Aranda y J.M. Salguero y R. Cabello (Coords.). Avances en el estudio de la inteligencia emocional (p. 563-568). Santander: Fundación Marcelino Botín.

Rizzolatti, G. y Sinigaglia, C. (2006). Las neuronas espejo. Barcelona: Paidós.

Rodríguez Garza, R. (2005). El desarrollo de la inteligencia emocional en niños de preescolar. Curso-taller del $5^{\circ}$ Encuentro Internacional de educación inicial y preescolar. Monterrey, mayo.

Ruiz de Velasco Gálvez, A. (2000). La dramatización como forma de desarrollar la inteligencia emocional. Indivisa: Boletín de estudios e investigación, 1, 191-196.

Salvucci, S., Walter, E., Conley, V., Fink, S. y Saba, M. (1997). Measurement error studies at the National Center for Education Statistics (NCES). Washington D.C.: U. S. Department of Education.

Sánchez Santamaría, J. (2010). La competencia emocional en la escuela: una propuesta de organización dimensional y criterial". ENSAYOS, Revista de la Facultad de Educación de Albacete, 25, 2010. Consultada el 19 de diciembre de 2012, en http://www.uclm.es/ab/educacion/ensayos.

Smith, W. J. (1977). The behaviour of communicating. Cambridge, M.A.: Harvard University Press.

Sotil, A., Escurra, L., Huerta, R., Rosas, M., Campos, E. y Llaños, A. (2008). Efectos de un programa para desarrollar la inteligencia emocional en alumnos de sexto grado de educación primaria. Revista de Investigación en Psicología, 11(2), 55-65.

Tomkins, S.S. (1963). Affect, imagery, consciousness. The negative affects. New York: Springer.

Torres, R.M. (2000). Una década de "Educación para Todos": La tarea pendiente. Madrid: Editorial Popular.

Weissberg, R.P. (2002). Collaborating to support the implementation of coordinated school-based prevention programming: Action steps and challenges. En P.J. Mrazek y C.M.H. Hosman (Eds.), Toward a strategy for worldwide action to promote mental health and prevent mental and behavioral disorders (pp. 46-48). Alexandria, VA: The World Federation for Mental Health.

Winston, J. (2000). Drama, Literacy and Moral Education 5-11. London: David Fulton.

Zillmann, D. (1994). Mechanisms of emocional involvement with drama. Poetics, 23, 33-51.

Fecha de recepción: 12 de diciembre de 2012.

Fecha de revisión: 13 de diciembre de 2012.

Fecha de aceptación: 21 de abril de 2013. 